\title{
Souvenirs d'une enfance douloureuse : l'expérience des enfants juifs cachés en Belgique
}

Adeline Fohn et Olivier Luminet

\section{(2) OpenEdition \\ 1 Journals}

Édition électronique

URL : http://journals.openedition.org/cmc/504

DOI : 10.4000/cmc.504

ISSN : 2684-3080

Éditeur

Fondation de la Mémoire Contemporaine

Édition imprimée

Date de publication : 1 décembre 2011

Pagination : 319-352

ISSN : 1377-1256

\section{Référence électronique}

Adeline Fohn et Olivier Luminet, «Souvenirs d'une enfance douloureuse : l'expérience des enfants juifs cachés en Belgique ", Les Cahiers de la Mémoire Contemporaine [En ligne], 10 | 2011, mis en ligne le 01 décembre 2019, consulté le 14 novembre 2020. URL : http://journals.openedition.org/cmc/504; DOI : https://doi.org/10.4000/cmc.504 


\title{
Souvenirs d'une enfance douloureuse : l'expérience des enfants juifs cachés en Belgique
}

\author{
Adeline Fohn - Olivier Luminet
}

En Belgique, de nombreux enfants juifs ont survécu aux persécutions nazies en se cachant ${ }^{1}$. Parmi ces enfants, certains ont été cachés sous une fausse identité et étaient "visibles" et forcés de mener une double vie à l'extérieur, tandis que d'autres étaient contraints à rester dans un endroit clos, à l'abri du regard de l'autre et non scolarisés². À partir de l'été 1942, les enfants juifs ont commencé à être placés dans des familles d'accueil ou dans un environnement institutionnel (couvents, orphelinats, maisons d'enfants). Dans la plupart des cas, les enfants cachés ont été séparés de leurs parents pour augmenter leur chance de survie. L'histoire d'Anne Frank et de sa famille, cachés ensemble dans une maison à Amsterdam, représente donc plutôt l'exception ${ }^{3}$. Tout au long de la guerre, les enfants juifs cachés ont dû faire face à des changements considérables : ils ont été séparés de leurs parents, ils ont dû vivre loin d'eux, souvent sans avoir de leurs nouvelles, coupés de leur culture; ils ont dû s'adapter à un milieu inconnu en cachant leurs origines, leur nom, voire l'existence de leurs parents ${ }^{4}$ Certains d'entre eux ont été convertis au catholicisme ${ }^{5}$.

\footnotetext{
${ }^{1}$ En Belgique, plus de la moitié de la population juive a échappé aux déportations (M. Steinberg, «L'enfant caché, le défi à la Shoah », dans I. Emery (Éd.), Histoire et mémoire des Juifs d'Anderlecht. Années 20-40, Bruxelles, 2009 ; Id., L'étoile et le fusil, 4 vol., Bruxelles, 1983-1986 ). Ida Sterno, l'une des responsables des enfants cachés du Comité de Défense des Juifs (CDJ), cite le chiffre de 2.293 enfants pris en charge et cachés par celui-ci, tandis que de nombreux parents ont souvent trouvé par eux-mêmes des relais indispensables pour placer leurs enfants avant d'être déportés ou d'entrer dans la clandestinité (M. Steinberg, ibid.; C. Massange, « Les homes d'enfants juifs à la Libération », dans Les Cahiers de la Mémoire contemporaine, $\mathbf{n}^{\circ}$ 9, 2010, pp. 59 . $88)$.

${ }^{2}$ D. Dwork, Children with a Star. Jewish Youth in Nazi Europe, New Haven \& London, 1991.

${ }^{3}$ D. Wolf, Beyond Anne Frank. Hidden children and Postwar Families in Holland, Berkeley, 2007.

${ }^{4}$ Voir M. Feldman, Entre trauma et protection. Quel devenir pour les enfants juifs cachés en France (1940-1944)?, Toulouse, 2009 ; A. Fohn, « Secret et traumatisme. L'expérience des enfants juifs cachés en Belgique ", dans L'autre: Clinique, Culture et Sociétés, nº 11 (2), 2010, pp.189-198; M. Frydman, Le traumatisme de l'enfant caché. Répercussions psychologiques à court et à long termes, Paris, 2002 ; A. Nysenholc, L'Enfant terrible de la littérature, Bruxelles, 2011 ; N. Zajde, « Le traumatisme des enfants cachés », dans Bulletin du Centre de Recherche français de Jérusalem, $\mathrm{n}^{\circ} 17,2006$, pp. 160-174.
} 
Certains enfants ont également souffert de malnutrition, de maltraitance ou ont été exploités.

La période d'après-guerre fut à la source de nouveaux traumatismes ${ }^{6}$. Les enfants ont appris la nouvelle de l'assassinat de leur(s) parent(s) ou ont été confrontés à des retrouvailles souvent traumatiques. Dans la majorité des cas, les parents survivants étaient "métamorphosés", profondément marqués par l'expérience concentrationnaire, la cache et la perte de leurs proches? ${ }^{7}$ Les enfants juifs cachés ont également vécu de nouvelles séparations en quittant le milieu qui les avaient accueillis pendant la guerre. Ces nouvelles séparations ont souvent ravivé la souffrance vécue lors de la séparation des parents, conférant une signification traumatique aux séparations ${ }^{8}$.

Après la guerre, les survivants ont tenté de se reconstruire en appréhendant l'avenir plutôt que de se tourner vers un passé trop douloureux. Jusque dans les années 1980, les enfants juifs cachés n’ont pas été reconnus comme survivants de la Shoah. Leurs voix étaient jusque-là absentes des grandes narrations du génocide ${ }^{9}$. Ils étaient considérés comme ayant eu "de la chance" en comparaison aux déportés et comme "trop jeunes" pour se souvenir du passé ${ }^{10}$. La plupart des enfants juifs cachés ont souvent tu leur histoire pendant près de quarante ans, voire soixante ans. Ce n'est qu'à partir de 1991, lors du premier grand rassemblement de nombreux anciens enfants juifs cachés à New York, que la plupart d'entre eux ont commencé à raconter leur histoire et qu'une réelle prise de conscience a eu lieu. La non-reconnaissance sociale est désignée comme l'un des facteurs permettant d'expliquer le temps de latence extraordinairement long

${ }^{5}$ F. Hogman, « The Experience of Catholicism for Jewish Children during World War II », dans Psychoanalytic Review, $\mathrm{n}^{\circ} 75$ (4), 1988, pp. 511-532.

${ }^{6}$ H. A. Keilson, Enfants victimes de la guerre, Paris, 1998.

${ }^{7}$ N. Zajde, Enfants de survivants. La transmission du traumatisme chez les enfants de Juifs survivants de l'extermination nazie, Paris, 2005 ; voir également Ead., Guérir de la Shoah, Paris, 2005.

8 A. Fohn - S. Heenen-Wolff, «The Destiny of an Unacknowledged Trauma. The Deferred Retroactive Effect of Après-coup in the Hidden Jewish Children of Wartime Belgium », dans International Journal of Psychoanalysis, $\mathbf{n}^{\circ} 92$ (1), 2011, pp. 5-20.

9 J. S. Kestenberg, « Memories from Early Childhood », dans Psychoanalytic Review, $\mathbf{n}^{\circ} 75$ (4), 1988, pp. 561-571.

10 J. Hefetz, Too Young to Remember, Detroit, 1989 ; M. Sternberg - M. Rosenbloom, « Lost Childhood. Lessons from the Holocaust : Implications for Adult Adjustment », dans Child and Adolescent Social Work Journal, $\mathbf{n}^{\circ} 17$ (1), 2000, pp. 5-17. 
avant que les enfants juifs ne se reconnaissent eux-mêmes comme ayant vécu un traumatisme.

Actuellement, il est incontestable que la majorité des enfants juifs cachés ont été confrontés à de nombreux événements traumatiques, pendant et après la guerre, dont les traces sont encore présentes actuellement (sentiment d'abandon, cauchemars, anxiété, processus de deuil différé, troubles de la filiation, troubles d'appartenance et de transmission $)^{11}$. Bien que le traumatisme des enfants juifs cachés soit de plus en plus étudié, aucune recherche scientifique n’a recensé de façon systématique les nombreux événements traumatiques vécus par ces enfants. Le but de la présente étude vise à mettre en évidence les souvenirs marquants, voire «potentiellement traumatiques », relatés par les enfants juifs cachés dans leurs récits. Nous pensons qu'il est possible de montrer qu'ils ont été confrontés à un « réel terrifiant » ${ }^{12}$. Notre travail consiste à étudier comment le vécu de cette population caractérise bel et bien une situation potentiellement traumatique. Pour chacun des récits, nous désirons mettre en évidence les divers éléments contextuels qui ont pu faire « effraction » dans la vie du sujet ${ }^{13}$.

\section{Méthode}

\section{Récolte des données}

Dans le cadre de notre recherche, nous avons récolté soixante récits de vie d'anciens enfants juifs cachés en Belgique qui ont survécu aux persécutions nazies ${ }^{14}$. Ces personnes ont pris contact avec Susann Hee-

11 E. Fogelman, « The Psychology behind being a hidden Child », dans J. Marks (Ed.), The Hidden Children. The Secret Survivors of the Holocaust, New York, 1993, pp. 292-307; M. Feldman, «Survie et destin psychique des enfants juifs cachés en France pendant la Deuxième Guerre Mondiale ", dans L'Autre, Cliniques, culture et sociétés, $\mathbf{n}^{\circ} 7$ (1), 2006, pp. 61-77; Y. Mouchenik, "Ce n'est qu'un nom sur une liste, mais c'est mon cimetière ». Traumas, deuils et transmission chez les enfants juifs cachés en France pendant l'Occupation, Grenoble, 2006 ; Id., « Traumas, deuils et témoignage. Élaboration des traumas et travail de deuil chez les enfants juifs cachés en France pendant l'Occupation », dans Revue francophone du Stress et du Trauma, $\mathrm{n}^{\circ} 10$ (3), 2010, pp. 155-161.

12 J. Altounian, La survivance. Traduire le trauma collectif, Paris, 2000.

${ }^{13}$ S. Freud, « Au-delà du principe de plaisir », dans S. Freud (éd. 2001), Essais de psychanalyse, Paris, 1920, pp. 47-128.

${ }^{14}$ Le présent article est rédigé dans le cadre d'une recherche doctorale entamée en septembre 2007 à l'Université de Louvain-la-Neuve (UCL, Belgique), financée par le Fonds spécial de Recherche 
nen-Wolff, psychanalyste et professeur à l'Université catholique de Louvain ainsi qu'à l'Université libre de Bruxelles, qui est à l'origine de ce projet de recherche universitaire. Les personnes intéressées par le fait de témoigner de leur histoire en tant qu'ancien enfant juif caché ont eu écho de la recherche par le bouche-à-oreille.

Les témoignages ont été recueillis selon la méthode du « récit de vie de recherche », permettant de parcourir l'histoire du sujet et de tenir compte de sa globalité, c'est-à-dire de sa naissance jusqu'au moment de l'entretien ${ }^{15}$. Le "récit de vie de recherche » peut être défini comme « un discours narratif qui s'efforce de raconter une histoire réelle et qui de plus, à la différence de l'autobiographie écrite, est improvisé au sein d'une relation dialogique avec un chercheur qui a d'emblée orienté l'entretien vers la description d'expériences pertinentes pour l'étude de son objet $»^{16}$.

Tout dispositif de recherche par le récit de vie se doit de suivre les étapes suivantes: (1) Le chercheur adresse une demande à un narrateur potentiel avec qui il prend contact et propose un contrat, (2) un ou plusieurs entretiens ont lieu et sont enregistrés sur magnétophone, (3) les entretiens sont retranscrits intégralement, (4) les entretiens font l'objet d'une analyse de la part du chercheur, (5) le processus s'achève par une publication ${ }^{17}$.

Généralement, un récit de vie comprenait deux à trois entretiens. La durée de chaque entretien variait entre une heure et demi et deux heures. Tous les récits ont été enregistrés avec l'accord des participants et le verbatim a été retranscrit. Les participants pouvaient nous recontacter s'ils étaient confrontés à des difficultés survenant dans le décours des entretiens. Un groupe de parole fut également mis en place entre octobre et décembre 2007 pour les sujets qui désiraient reparler de leur vécu. Tout comme d'autres auteurs qui ont travaillé dans un contexte comparable, nous avons abordé avec les enfants cachés les

(FSR). Dans cette recherche, nous essayons de comprendre l'organisation psychique spécifique de ces enfants devenus adultes afin de comprendre l'impact à long terme d'un traumatisme collectif précoce, longtemps non reconnu.

${ }^{15}$ M. Legrand, L'approche biographique. Théorie, clinique, Paris, 1993 ; D. Bertaux, L'enquête et ses méthodes. Le récit de vie, Paris, 2005.

${ }^{16}$ D. Bertaux, op. cit., p. 68.

${ }^{17}$ M. Legrand, op. cit., p. 184. 
périodes d'avant, pendant et après la guerre ${ }^{18}$. Nous avons laissé les personnes rencontrées aborder librement l'histoire de leur vie et les événements qu'elles jugent importants. Ensuite, nous leur avons demandé d'approfondir certains moments de vie ou d'apporter des précisions supplémentaires.

\section{Participants}

Suite à notre demande, les participants se sont portés volontaires pour témoigner de leur histoire. Quarante récits de vie ont été analysés dans le cadre de cette recherche. En 1942, les personnes que nous avons interviewées étaient âgées de quelques mois à 12 ans. La moyenne d'âge était de 6 ans et demi. Au moment de l'interview, ils avaient entre 65 et 80 ans. La moyenne d'âge était de 73 ans. Dix-huit étaient des femmes et vingt-deux des hommes. Tous sont nés en Belgique, excepté deux personnes (l'une est née en Autriche, l'autre en Allemagne). Une grande majorité des sujets provenaient de familles juives, principalement d'origine polonaise, qui ont immigré en Belgique dans les années 20-30. Si certaines avaient obtenu la nationalité belge, la plupart d'entre elles étaient apatrides ${ }^{19}$. La langue maternelle des participants était le yiddish, le français ou l'allemand. Tous proviennent de milieux urbains et sont principalement issus des villes d'Anvers et de Bruxelles. Une minorité d'entre eux vit à Liège et à Charleroi.

\section{Analyse des données}

D'un point de vue méthodologique, nous avons procédé à une analyse qualitative transversale des récits afin de mettre en évidence les souvenirs liés aux événements traumatiques de guerre et

\footnotetext{
${ }_{18}$ Voir J. S. Kestenberg, op. cit., pp. 561-571 ; H. A. Keilson, Enfants victimes de la guerre, Paris, 1998 ; M. Feldman, Psychologie et psychopathologie des enfants juifs cachés en France pendant la Seconde Guerre Mondiale et restés en France depuis la Libération, thèse en psychologie, Paris, Université Paris 13, 2007 ; S. Kaplan, Children in Genocide. Extreme Traumatization and Affect Regulation, Londres, 2008.

${ }^{19}$ En Belgique, une petite minorité des Juifs étaient belges : M. Steinberg, L'Étoile et le fusil. La traque des Juifs : 1942-1944., vol. 1, Bruxelles, 1986 ; S. Brachfeld, Ils ont survécu. Le sauvetage des Juifs en Belgique occupée, Bruxelles, 2001.
} 
d'immédiat après-guerre. Récit après récit, les extraits relatifs à des souvenirs potentiellement traumatiques ont été repérés, annotés par le chercheur et codés dans le logiciel d'analyse thématique Nvivo $7^{20}$. Au total, quarante récits ont été choisis aléatoirement et codés dans le logiciel. Le codage des deux-tiers des récits récoltés nous a permis d'obtenir des informations représentatives de l'échantillon et nous avions obtenu un point de saturation: aucun nouveau récit ne permettait de dégager de nouvelles catégories relatives aux souvenirs potentiellement traumatiques dans les récits ${ }^{21}$. Nous avons ensuite parcouru une nouvelle fois les autres récits non codés afin de s'assurer qu'aucune autre catégorie n'émergeait. Une situation particulière et peu rapportée dans les autres récits, à savoir l'abus sexuel, a été repérée et ajoutée dans le travail de description. Nous avons ensuite procédé à une vérification du codage auprès d'Olivier Luminet, au vu de ses connaissances théoriques sur la mémoire. Cette procédure a permis de valider notre dispositif et de réduire le biais de subjectivité du chercheur ${ }^{22}$. Enfin, avec l'aide de ce même tiers, nous avons procédé à une sous-catégorisation des données afin de structurer et d'organiser les données en vue de leur présentation ${ }^{23}$.

\section{Présentation des résultats}

L'analyse des données nous a permis de dégager quatre catégories de souvenirs traumatiques que nous allons présenter: les souvenirs relatifs à l'atteinte identitaire, à la crainte d'être découvert, aux violences et aux pertes.

\section{Les souvenirs liés à l'atteinte identitaire}

Parmi les souvenirs liés à l'atteinte identitaire, nous avons dégagé le port de l'étoile, le retrait de l'école, les remarques péjoratives et les agressions liées à l'appartenance juive, le changement de nom, la conversion catholique et le danger lié à la circoncision.

${ }^{20}$ G. R. Gibbs, Qualitative Data Analysis. Explorations with NVivo, Berkshire, UK, 2002.

${ }^{21}$ M. Legrand, op. cit., Paris, 1993 ; G. R. Gibbs, op. cit.

${ }^{22}$ M. Santiago-Delefosse, Les méthodes qualitatives en psychologie, Paris, 2001.

${ }^{23}$ G. R. Gibbs, op.cit 
En Belgique, à partir du 7 juin 1942, suite à l'ordonnance du 27 mai 1942, le port de l'étoile de David fut obligatoire pour toute personne juive de plus de 6 ans $^{24}$. Le port de l'étoile s'inscrivait dans le cadre de la «solution finale », la destruction totale de tous les Juifs d'Europe. Parmi les personnes interviewées qui avaient plus de 6 ans (24 personnes), 13 enfants ont porté l'étoile tandis que onze d'entre eux ne l'ont pas portée. Ces chiffres attestent qu'une partie des parents étaient conscients du danger et refusaient que leurs enfants portent ce signe distinctif. Toutes les personnes ayant porté l'étoile en ont parlé dans leur récit. Ce souvenir, lié à l'exclusion sociale, reste très marquant. Madame B., née en 1930, rapporte un événement douloureux et angoissant lié au port de l'étoile: «J'avais une étoile juive sur mon manteau et mes parents, par prudence, disaient : "On ne sait pas ce qu'il peut se passer. Tu retournes ton manteau avec ton étoile à l'intérieur et tu le portes sur le bras". Alors je me suis présentée à cet examen d'entrée avec autour de mon bras ce manteau entouré et une compagne de classe qui le présentait en même temps que moi a dit à toutes les autres qui étaient là : "Vous savez pourquoi elle porte son manteau sur le bras, roulé comme ça ? Parce qu'elle est juive et parce qu'elle porte l'étoile". Enfin vous imaginez ce que ça a fait. Et ça m'a fait d'autant plus mal que c'était la fille d'une institutrice de l'école. Et à part une ou deux amies, j'ai à peu près oublié le nom de toutes mes compagnes de classe. Mais son nom à elle, je l'ai vraiment bien gravé dans mon esprit. »

Le retrait scolaire représente également un événement particulièrement important pour les enfants juifs scolarisés. À partir du 31 décembre 1941, l'exclusion scolaire concernait les enfants juifs qui n'étaient pas en âge d'obligation scolaire (enseignement maternel et cycle secondaire supérieur). Å partir de septembre 1942, l'exclusion scolaire concernait tous les enfants juifs, y compris les enfants en âge d'être scolarisés ${ }^{25}$. Le retrait de l'école représente une nouvelle atteinte identitaire. Monsieur B., né en 1928, se souvient d'avoir vécu le retrait du milieu scolaire comme un événement très humiliant. Madame R. se souvient d'avoir vécu un sentiment d'injustice et d'incompréhension :

${ }^{24}$ M. Steinberg, L'Étoile...,op. cit.

${ }^{25}$ Ibid.; V. Teitelbaum-Hirsch, Les larmes sous le masque, Bruxelles, 1994; B. Dickschen, L'école en sursis. La scolarisation des enfants juifs pendant la guerre, Bruxelles, 2006. 
« Un beau jour, en 1942, je suis rentrée de l'école en disant à mon papa: “Tu sais, il y a une affiche qu'on a mise dans le hall de l'école. Les enfants juifs ne pourront plus venir à l'école. C'est quoi un enfant juif ?" Alors, il m'a dit: "Écoute, c'est un peu compliqué de t'expliquer ça maintenant, mais toi aussi, tu es un enfant juif et tu n'iras plus à l'école à partir de ce jour-là". Imaginez la crise! Pourquoi? Qu'est-ce que j'ai fait? Pourquoi est-ce que je suis maudite? Pourquoi est-ce que je ne peux plus aller à l'école? C'est la première incompréhension, la première fois que j'étais confrontée à une injustice que je ne comprenais pas. »

Les remarques antisémites et les agressions liées à l'appartenance juive sont venues fragiliser la construction narcissique-identitaire des sujets. Les enfants juifs qui portaient l'étoile étaient particulièrement exposés à des insultes antisémites, et parfois victimes d'agressions physiques. L'appartenance juive était une fois de plus pointée du doigt et méprisée, à tel point que l'enfant tentait souvent de cacher ce signe distinctif : « Pour aller à l'école, j'avais cette étoile sur mon veston. Il y avait donc évidemment les réflexions des copains: "Tiens, tu es juif, toi! Je ne savais pas que tu étais juif ". Mais bon, c'était très amusant aux yeux des autres mais ça m'ennuyait beaucoup... à tel point qu'en rue, j'essayais de camoufler l'étoile derrière mon cartable pour qu'on ne la voie pas. Il pouvait arriver que des antisémites puissent m'agresser puisque j'avais l'étoile. » (Monsieur B.)

De façon générale, l'antisémitisme est venu fragiliser l'enfant en pleine construction. Madame $\mathrm{Ch}$. met en évidence le conflit interne contre lequel elle a dû lutter tout au long de sa vie : « Il en résulte un tiraillement persistant entre deux vues contradictoires de moi-même : d'une part, une vermine-par-nature-destinée-à-l'anéantissement, puisque c'était ça que disaient les adultes quand j'avais 7 ans, et d'autre part, une enfant qui savait lire à 4 ans, qui a un QI de 150 , une vaste culture, qui connaît sept ou huit langues et a fait plusieurs fois le tour du monde. Pas facile à concilier. »

Tout au long de leur vie, ils ont souvent continué à se "cacher" et ont tenté de trouver de nouvelles appartenances pour fuir le poids et le danger intériorisé en lien avec l'appartenance juive. La charge émotionnelle est souvent forte lorsque l'atteinte identitaire est abordée dans les récits. Lorsque monsieur P., né en 1932, parle de ce qu'il a 
vécu, il retient particulièrement le fait d'avoir été rabaissé et la déshumanisation : "Chez nous autres, juifs, quand on est insulté... c'est toujours très pénible [silence]. C'est comme les gens... n'ou... n'oubl... n'oublir... n'ou... n'oublierons jamais les nazis [silence], parce que c'était vraiment... rabaisser l'être humain à les... à la bête. »

Actuellement, les insultes antisémites lui font repenser au bruit de bottes des nazis. La remémoration de ces moments traumatiques le renvoie à des éléments sensoriels marquants, toujours très présents actuellement. Le rappel d'éléments sensoriels est lié à des souvenirs particulièrement vivaces et souvent traumatiques. Aujourd'hui, monsieur $P$. ne parle pratiquement pas de son vécu qui reste douloureux à aborder : « J'en parle très peu. La réaction est toujours la même... Maintenant, que je vous ai parlé, j'ai les larmes aux yeux [il se contient]. Ça me donne beaucoup d'émotions. Je pense qu'on a été humilié comme si on était des bêtes humaines. Je sais que quand j'étais un enfant, j'avais peur [silence]. Une terrible peur... »

La peur est toujours présente chez la plupart des personnes que nous avons rencontrées. Elles craignent le retour de violences antisémites et que leurs enfants et petits-enfants soient un jour confrontés à de telles expériences.

Le changement de nom, vécu par la majorité des personnes que nous avons rencontrées, a souvent contribué à une atteinte identitaire et à une atteinte de la filiation. Madame R. se souvient : « Il fallait entrer dans la peau de quelqu'un d'autre. À un moment, en 1942, j'ai cessé d'être moi pour devenir quelqu'un d'autre. Et au bout de deux ans, j'ai de nouveau dû changer, cesser d'être ce quelqu'un d'autre pour enfin devenir moi. Mais est-ce que le moi que j'étais devenue était le même que celui que j'avais abandonné ? Ça, je ne le saurais jamais. » Cette transformation, nécessaire pour survivre, est par la suite venue créer une confusion identitaire chez de nombreux enfants juifs cachés. Comment se construire sur base d'une double identité ? Qui suis-je ? Que serais-je devenu(e) si je n'avais pas changé de nom, si je n'avais pas été séparé de mes parents ? Ces questions sont très présentes chez les anciens enfants juifs cachés.

La conversion au catholicisme représente également une expérience importante qui est venue couper un peu plus les enfants cachés de leur milieu d'origine. Dans notre population, dix-neuf personnes ont été 
cachées dans un environnement catholique pratiquant. Certains ont été baptisés, certains ont fait leur communion et se rendaient à l'église, apprenant les prières et des chants religieux. Comme beaucoup d'autres, madame St., née en 1929, fut cachée dans un couvent et marquée par l'idéologie catholique : "Je ne connaissais que la prière du Vendredi Saint: "Prions pour les perfides juifs" qui me faisait beaucoup de mal. Je sais qu'un des gros traumatismes que j'ai eu, c'est qu'au cours de religion, quand on disait : "Que son sang retombe sur nos têtes", parce que les Juifs avaient commis un déicide, là, je me rappelle, je me serais cachée en-dessous d'un banc et je me sentais terriblement mal à l'aise parce que j'avais l'impression d'être la coupable au sein de tout ce monde d'enfants catholiques qui étaient très, très gentils parce qu'ils n'ont jamais fait de racisme, jamais, jamais, jamais!»

Comme le souligne Hogman, pendant la guerre, les croyances catholiques ont parfois aidé les enfants juifs à faire face au manque parental, lui donnant l'impression d'être accepté, rassuré, protégé du monde extérieur et des humiliations ${ }^{26}$. Après la guerre, ils ont dû se réadapter au milieu juif, ce qui fut d'autant plus difficile lorsque les enfants avaient été convertis au catholicisme. Certains ont continué à se rendre à l'église, d'autres en ont voulu à leurs parents survivants de les avoir empêché de faire leur communion, d'autres encore se sont mariés à un conjoint catholique. Nous constatons fréquemment que les orphelins ont souvent éprouvé plus de difficultés à se tourner vers le milieu juif après la guerre, particulièrement ceux qui n'ont pas été élevés par la suite dans une institution juive. De plus, la conversion religieuse a davantage suscité un sentiment de culpabilité et de trahison chez les orphelins, comme le souligne madame St. : « J'étais très culpabilisée de ma conversion parce que je me suis dit, en me convertissant, je réalise exactement ce que les nazis ont voulu faire, c'est-à-dire supprimer les Juifs ». Le catholicisme est parfois venu créer une confusion et un sentiment de double appartenance. Monsieur Z., orphelin de la Shoah, se souvient avoir été enfant de chœur durant la

${ }^{26}$ F. Hogman, « The Experience of Catholicism for Jewish Children during World War II », dans Psychoanalytic Review, $\mathrm{n}^{\circ} 75$ (4), 1988, pp. 511-532; voir également S. Vromen, Hidden Children of the Holocaust. Belgian Nuns and their Daring Rescue of Young Jews from the Nazis, Oxford, 2008 
guerre. À l'âge adulte, il s'est marié avec une femme catholique pratiquante. Il souligne actuellement qu'il connaît mieux les prières catholiques que juives.

La circoncision chez les garçons représentait un danger supplémentaire d'être découvert. La circoncision des garçons révélait irréfutablement leur judéité. Certains garçons ont parfois été habillés comme des filles durant la guerre pour éviter les contrôles physiques ${ }^{27}$. Les souvenirs, liés à la circoncision et vécus avec angoisse au moment de la guerre, sont aujourd'hui encore décrits avec une grande précision : " Il y a certainement eu une dénonciation, parce que nous avons eu la visite de la Gestapo. Un Belge et un Allemand. Je ne l'oublierai jamais parce que cela m'a vraiment choquée, en ce sens qu'ils sont venus et mon frère, qui était déjà un grand garçon, il a six ans de plus que moi... je n'oublierai jamais ce geste, l'Allemand qui a pris mon frère par ici et qui a dit: "Du bist jude" [tu es juif]. Et les personnes chez qui nous étions ont dit: “Non, non, c'est mon petit filleul". Heureusement qu'ils n'ont pas fait baisser le pantalon. »

Ces événements traumatiques liés au risque de mort ont parfois influencé certaines familles juives à renoncer à la circoncision. Madame St. souligne: «J'avais l'impression que je ne pouvais plus les [ses enfants] charger d'une marque ». Madame F. a perdu son frère, déporté et assassiné à Auschwitz suite à un contrôle de la Gestapo qui constata sa circoncision. Son père refusa de faire circoncire son fils, né après la guerre, par crainte que de telles atrocités ne se reproduisent. Ces événements, qui ont marqué la mémoire individuelle et collective du peuple juif, ont parfois amené certaines personnes à repousser l'appartenance juive: "Pendant des années, pour moi cela a été comme un rejet, cela a pesé trop lourd dans ma vie » (Madame F.). Si le rejet de l'appartenance juive intervient pour protéger le sujet de nouvelles souffrances, ce rejet est souvent lié à long terme à une souffrance identitaire et à un sentiment de solitude important.

\footnotetext{
${ }^{27}$ M. Sternberg - M. Rosenbloom, op. cit., pp. 5-17 ; M. Kestenberg - J. S. Kestenberg, « The Sense of Belonging and Altruism in Children who survived the Holocaust », dans Psychoanalytic Review, 75(4), 1988, pp. 533-560.
} 


\section{Les souvenirs liés à la crainte d'être découvert}

Tout au long de la période de la clandestinité, la majorité des enfants juifs cachés craignaient d'être découverts. Seuls les jeunes enfants qui n'avaient pas compris le danger n'ont pas éprouvé cette crainte permanente. Parmi les souvenirs liés à la crainte d'être découverts, nous avons dégagé le risque d'être pris, les menaces de dénonciation et les dénonciations, les rafles, le contact avec les Allemands et la crainte du retour des Allemands.

Le risque d'être pris était déjà présent dès le début de la guerre, au moment de l'exode. Après l'invasion de la Belgique par les Allemands le 10 mai 1940, de nombreuses familles juives ont quitté la Belgique pour la France, espérant rejoindre des zones non occupées. Par la suite, le passage de la zone occupée à la zone libre comporta toujours des risques importants. Madame S., âgée de 4 ans en 1940, se souvient: « J'ai toujours été une petite bronchiteuse et mon père me disait en yiddish : "Tu ne tousses pas, on va passer là, on va passer là derrière avec le passeur mais je t'interdis de tousser parce qu'on va se faire remarquer, et on va tous se faire prendre." Bon la culpabilité, elle a commencé là [rires]. Ça c'est une évidence. Bref, je n'ai pas toussé puisqu'on est passé. Mais je me souviens très bien de la menace parce que d'autres ont suivi. C'était le début des menaces et le début de la culpabilité aussi. Si jamais je tousse, pff... ce sera de ma faute. C'est mon premier souvenir. »

D'autres enfants, qui ne se sont pas retrouvés dans des situations aussi risquées et qui se sont sentis protégés par leurs parents, ne se sont parfois pas rendu compte du danger. Par exemple, madame Ch. et monsieur $R$. ont vécu l'exode comme un voyage, une "aventure extraordinaire $"$.

Les menaces de dénonciation, voire les dénonciations effectives, ont souvent été relevées dans les récits des enfants juifs cachés. Durant la période de cache, monsieur A., né en 1935, se souvient qu'il fut brutalement retiré de l'école qu'il fréquentait sous un nom d'emprunt suite à une menace de dénonciation : « J'ai commencé à aller à l'école mais pas très longtemps parce que, dans une bagarre d'enfants de l'école sur le chemin du retour, un gosse m'a dit: "Mais on sait très bien que tu es juif, on va te dénoncer". Donc on ne m'a plus mis à 
l'école, j'ai donc passé deux ans sans aller à l'école. C'est un souvenir marquant. »

De nombreux enfants ont également été dénoncés, précipitant un départ inévitable pour survivre. Le fait d'avoir souvent changé de lieu de cache pendant la guerre est lié à une perception du danger plus importante chez l'enfant car ces changements ont souvent eu lieu dans la précipitation et dans l'angoisse. Les cauchemars des enfants juifs cachés traduisent encore la crainte d'être découvert, l'enfermement ou l'impossibilité de s'échapper.

Les rafles sont également liées à des souvenirs d'une grande précision après plus de 65 ans. En Belgique, quatre grandes rafles ont eu lieu à Bruxelles et à Anvers durant l'été 1942, du 15 août au 11 septembre ${ }^{28}$. Les rafles avaient généralement lieu la nuit (les nuits du 15 août, du 28 août et du 3 septembre 1942). La quatrième rafle diffère des autres car elle a eu lieu durant deux journées consécutives les 11 et 12 septembre 1942. À partir de septembre 1943, les nazis s'en sont également pris aux Juifs de nationalité belge. Une nouvelle grande rafle a eu lieu à Anvers et surtout à Bruxelles dans la nuit du 3 au 4 septembre 1943. Près de la moitié des personnes interviewées ont parlé des rafles dans leur récit. Lorsque les rafles ont débuté en aoûtseptembre 1942, de nombreux enfants n'étaient pas encore cachés. C'est souvent à ce moment-là que les familles juives ont pris conscience de l'urgence d'entrer dans la clandestinité. La panique et l'angoisse sont encore perceptibles dans les récits comme en témoigne madame Ga. : "Avant d'être cachée, c'était la panique! Ma mère... on nous a montré dans... Au grenier, il y avait une espèce de faux mur. D'après mon souvenir, une espèce de cloison, il fallait grimper la cloison. Ma sœur et moi, je devais l'entraîner derrière la cloison, ils auraient enlevé le tabouret ou je-ne-sais-quoi pour aller derrière la cloison, mes parents, ils se seraient donnés aux Allemands... enfin, quand ils auraient été pris, nous, on... je devais prendre ma sœur par la main et aller, je me rappelle, derrière... on m'expliquait le chemin, chez Mademoiselle Duchamp. Je me souviens encore du nom. »

Monsieur T., âgé de 6 ans, se souvient quant à lui de s'être caché avec sa mère, à plat ventre dans le jardin, pour échapper aux

${ }^{28}$ M. Steinberg, L'Étoile et le fusil. 1942, Les cent jours de la déportation des Juifs de Belgique, Bruxelles, 1984. 
Allemands : « J'ai vu mon père entouré de soldats. Ils ont cherché. Heureusement, ils n'avaient pas leurs chiens, ils ne nous ont pas vus... Ma mère tenait sa main sur ma tête pour être sûre que je ne la lève pas... Ils sont partis, et la première nuit qui a suivi, on m'a mis sur un vélo et on m'a mis dans cette famille. »

Les rafles ont également parfois eu lieu dans la famille ou l'institution qui cachaient l'enfant. Dans notre population, nous avons constaté que les rafles étaient plus fréquentes dans les institutions qu'en famille d'accueil, ce qui peut s'expliquer par le nombre plus important d'enfants cachés et la démoniaque politique nazie, qui vérifiait scrupuleusement si les institutions cachaient des enfants. Monsieur E., né en 1933, a échappé de justesse à une rafle dont la majorité des enfants fut déportée. Sa réaction traduit l'effondrement qui s'est produit après cet événement : « Je suis sorti, on a tourné et là, je me suis effondré. Je me suis mis à trembler. Je me rappelle très bien, je savais plus bouger. Je me rappelle très bien. Je me sens tellement loin de cet enfant, mais de ça je m'en rappelle. » Lors de son récit, il semblait revivre l'événement. Ses souvenirs sont extrêmement précis et font intervenir de nombreux éléments sensoriels, visuels ou auditifs (le bruit des bottes, une phrase prononcée, le visage de l'Allemand).

Durant la période de cache, certaines personnes se souviennent d'avoir été pétrifiées suite à un contact avec un Allemand. Monsieur H. souligne : « Les Allemands m'ont tapoté la joue ou bien ils faisaient des plaisanteries. Moi, ils me terrifiaient. J'avais vraiment une peur panique des Allemands. On voyait que j'étais terrifié, alors ils me donnaient un bonbon pour apparaître moins terrifiants. " Certains enfants ont également vécu quotidiennement à proximité des Allemands, ce qui était à la source d'une angoisse permanente.

Enfin, dans leur récit, de nombreuses personnes ont parlé de l'offensive von Rundstedt qui a eu lieu le 16 décembre 1944 alors que la Belgique avait été libérée trois mois plus tôt ${ }^{29}$. Cette nouvelle invasion est liée à la réémergence de craintes importantes : «Je suis toujours restée angoissée... Et puis il ne faut pas oublier que quand on

${ }^{29}$ L'offensive von Rundstedt est encore appelée « la Bataille des Ardennes ». Elle a commencé le 16 décembre 1944 et s'est achevée en janvier 1945. Alors que la Libération survenait en septembre 1944, les Allemands ont attaqué par surprise l'Ardenne belge (sud de la Belgique) et le nord du Luxembourg. 
est rentré à la maison, je suis rentrée quelques semaines après, et il y a eu l'offensive von Rundstedt. C'était de nouveau la pagaille, c'était de nouveau... Je voyais les gens dans la rue qui se parlaient, qui disaient [angoisse, accélération du débit de parole]: “Mais qu'est-ce qu'il faut faire ? Il faut de nouveau fuir si jamais les Boches reviennent, il faut de nouveau partir, ça va de nouveau recommencer" [silence]. » (Madame Fr.)

Les conséquences des peurs intenses vécues durant la guerre ont laissé des traces psychiques indélébiles. À l'âge adulte, nombreux sont ceux qui ne voulaient pas se rendre en Allemagne, acheter une voiture de marque allemande ou entendre l'allemand. Monsieur H. souligne : « J'ai mis du temps avant de pouvoir traverser l'Allemagne, la langue allemande est pour moi terrifiante ». Madame Fr. souligne : « Je vais vous dire qu'aujourd'hui encore, je tremble pour mes enfants et mes petits-enfants. Même maintenant, j'ai peur pour eux. J'ai peur pour eux parce que ils sont jeunes, que je ne supporterais pas que mes petits-enfants doivent encore subir des insultes, qu'on doive les... les... les toucher, les frapper, je ne... je ne le... je ne le supporterais pas. Je ne le supporterais pas. "Presque toutes les personnes rencontrées craignent le retour possible d'hostilités et ne se sentent jamais en sécurité. Les massacres de masse ultérieurs à la Shoah (Rwanda, ExYougoslavie, Darfour) ou le conflit israélo-palestinien les marquent considérablement et favorisent la remémoration de leur vécu traumatique.

\section{Les souvenirs liés aux violences}

Parmi les souvenirs liés à la brutalité, nous avons relevé la violence envers les Juifs, le fait d'avoir été témoin de scènes de mort, l'internement dans un camp de rassemblement, les bombardements, la maltraitance dans la famille d'accueil, le retour des déportés, la découverte du massacre et les retrouvailles traumatiques avec les parents.

Certaines personnes ont assisté à des scènes particulièrement violentes envers des Juifs, spécifiquement deux personnes venues d'Autriche et d'Allemagne, ainsi qu'une personne à Anvers. Madame St. est née en Autriche en 1929 et a fui son pays natal dans la précipitation. Elle se 
souvient d'une enfance sereine qui trouva une fin abrupte au moment de l'Anschluss ${ }^{30}$. Elle fut alors confrontée à des scènes terrifiantes: «Cela a été d'une sauvagerie incroyable, les Juifs qui étaient pratiquants et qui portaient la barbe et, comment on appelle cela... des tresses... oui, des papillotes. J'en ai vu qu'on a tiré par la barbe hors de leur maison et qu'on leur coupait la barbe avec des morceaux de menton. Enfin, c'était... Je n'aime pas trop parler de cela parce que c'est le moment le plus sombre de mon enfance. »

Une personne nous a également parlé du «pogrom d'Anvers », qui s'est déroulé le 14 avril 1941 dans le quartier juif ${ }^{31}$. Le quartier fut attaqué par des sympathisants de l'occupant qui appartenaient à la SS flamande, la brigade noire et le Vlaams Nationaal Verbond (VNV), le parti nationaliste flamand collaborationniste. Monsieur Su., âgé de 4 ans et demi ans lors des faits, se souvient de cet événement : « Je me souviens, m'être promené main dans la main avec mon père, avec un petit gamin, et nous allions dans la rue de l'Orient. Je l'ai noté ici, en flamand, c'est Oostenstraat, et je voyais les Allemands sortir les bancs, les tables, les chaises, les rouleaux de la Bible, ils ont tout jeté au milieu de la rue, ils en ont fait un énorme brasier, donc ils ont allumé tout ça, ça m'a vraiment fait un choc. J'avais peur! Alors ils ont cassé les vitraux. Il y avait de très beaux vitraux, des mosaïques qu'ils ont cassés. »

Nous constatons une fois de plus que les violences vécues restent gravées dans la mémoire des anciens enfants juifs cachés, et ce même chez les plus jeunes. Dans leur enfance, ils ont intériorisé que l'appartenance juive est liée au danger, à l'insécurité ${ }^{32}$. Le traumatisme se produit alors que ces enfants ne se sont pas encore construit une personnalité stable et mature ${ }^{33}$. Contrairement aux adultes, ils ne peuvent pas s'appuyer sur des expériences de vie pré-traumatique paisibles et apaisantes qui pourraient les aider à supporter les événements auxquels ils ont dî faire face.

${ }^{30}$ L'annexion de l'Autriche par l'Allemagne nazie a eu lieu le 12 mars 1938.

${ }^{31}$ Comme le souligne S. Brachfeld (op. cit.), il s'agissait d'une action de terreur dans laquelle deux synagogues ainsi que les rouleaux de la Torah et les livres de prière ont été brûlés.

${ }^{32}$ A. Fohn, « Secret et traumatisme... », op. cit., pp. 189-198.

${ }^{33}$ H. Dasberg, « Late-onset of post-traumatic Reactions in Holocaust Survivors at advanced Age », dans H. Rossberg - J. Lansen (éds.), Das Schweigen Brechen (Breaking the Silence), Berlin - New York, 2003, pp. 311-348. 
Dans notre population, quatre personnes ont été confrontées à la mort dans des circonstances différentes (exode, arrestation, libération). Âgé de 4 ans et demi, monsieur Fu. se souvient avoir vu des Allemands tirer sur un homme : « Ma mère était venue me chercher un jour, elle m'avait emmené quelque part, voir un type qui imitait Charlie Chaplin. Quand on est sorti, on a descendu une rue et on a vu des casques, là dans le fond. Alors on a entendu : "Feuer!" et il y avait un type à côté de nous qui est tombé. Et on est descendu la rue, et il y avait un groupe d'Allemands qui était là avec d'autres gens qui avaient été faits prisonniers et qui nous ont dit: "Foutez le camp ! Foutez le camp!". Ça, c'est un événement, quand on est enfant, qui forcément... » Malgré son jeune âge, cet événement reste gravé dans sa mémoire. Il lui arrive d'y repenser encore aujourd'hui. Madame F. a elle aussi côtoyé la mort et y a échappé de justesse : «Nous avons été mitraillés sur les routes par l'aviation allemande. On a failli mourir plus d'une fois. Ils mitraillaient sur les routes tout ce qui bougeait et on se jetait dans les fossés. Papa et maman au-dessus de nous pour nous protéger. Écoutez, un enfant de huit ans qui voit des gens se faire mitrailler autour de soi [soupir]... Je crois que je n'ai jamais osé parler de ça à mes petits-enfants pour ne pas les traumatiser. C' est une vision atroce, atroce. »

La majorité des anciens enfants juifs cachés ont gardé ces souvenirs traumatiques pour eux, sans pouvoir les partager et en parler avec autrui. En vieillissant, ils soulignent que le passé refait surface et qu'ils se sentent plus vulnérables, plus fragiles qu'avant.

Dans notre population, quatre personnes ont été internées dans un camp de rassemblement avant d'être cachées. Ces enfants ont été sauvés de justesse de la déportation. Cette expérience, peu relatée dans la littérature, fait pourtant partie de celle de certains enfants juifs cachés. Monsieur B. a été arrêté avec ses parents à l'âge de deux ans et interné à la caserne Dossin pendant dix mois ${ }^{34}$. Ses souvenirs font principalement intervenir des éléments sensoriels : «Il me reste

${ }^{34}$ Lieu de rassemblement des Juifs en Belgique avant d'être déportés vers Auschwitz. Comme le souligne S. Brachfeld (op. cit., p. 51), un certain nombre de Juifs belges avaient été arrêtés lors des razzias à Bruxelles et à Anvers et étaient enfermés à la caserne Dossin sans être envoyés à Auschwitz; ce qui était le cas de la famille de monsieur B. Les 26 et 29 juin 1943, environ 200 d'entre eux ont été libérés de Malines. Après avoir été libérés, monsieur B. et sa famille sont entrés dans la clandestinité et ont survécu à la guerre en se cachant. 
quelques vagues images d'une grande chambre avec des paillasses par terre et comme je suis petit, je vois, je me souviens, de grandes bottes qui passaient près de moi, c'était les bottes des Allemands. Je me souviens de chiens qui aboient, je me souviens... mais, vous savez à 3 ans, j'allais avoir 3 ans, donc c'est vraiment, ça doit être très, très vague, mais il y a des images. Je vois quelqu'un qui me donne dans cette énorme pièce où il n'y a que des paillasses... je vois quelqu'un qui me donne encore une poupée, un petit chien que j'ai gardé longtemps. » (Monsieur B., né en 1940).

Trois personnes ont été internées en France dans le camp de Rivesaltes ${ }^{35}$. Monsieur Sy., né en 1933, y est resté pendant un an et demi avec sa mère. Il se souvient de la violence entre les enfants, attisée par les gardiens du camp. Cette confrontation à l'inhumanité le bouleverse encore aujourd'hui : «À Rivesaltes, les gardiens excitaient les petits Juifs contre les petits Espagnols pour qu'ils se battent ensemble. Il m'a fallu quarante ans pour comprendre que ça les faisait jouir ces gens de nous voir nous battre! Et je suis incapable de comprendre pourquoi! [Il se met à pleurer.] C'est l'incompréhension, ça me dépasse ! Où était leur plaisir ?»

Plus de la moitié des personnes interviewées ont également parlé des bombardements auxquels ils ont été exposés, notamment quand furent lancés les $\mathrm{V} 1$ et les $\mathrm{V} 2^{36}$. Ces événements, sont liés à un sentiment de peur intense : « Il y a eu ce bombardement sur la gare d'Ottignies, qui était un terrible bombardement d'autant plus qu'ils jetaient les bombes non pas seulement sur la gare, mais les Américains, ils jettent les bombes un peu partout [rire]. Ça et là ! J'étais très, très angoissé. Ça, je m'en souviens et alors, je priais pour que les avions qui passaient au-dessus ne me tombent pas dessus. » (Monsieur A.) ${ }^{37}$.

Certains enfants juifs cachés ont aussi vécu des violences au sein du milieu d'accueil. Dans leur récit, cinq personnes ont rapporté des faits

${ }^{35}$ Il s'agit d'un camp de rassemblement et de transit essentiellement pour les Juifs situé près de Perpignan (France).

${ }^{36}$ Il s'agit de bombes volantes qui étaient utilisées par l'Allemagne nazie à la fin de la guerre.

${ }^{37}$ Entre mars et début juin 1944, les Alliés préparaient le débarquement de Normandie (6 juin 1944) et attaquaient le réseau ferroviaire afin de gêner le déplacement des convois de l'armée allemande (opération Railfree). Ils ont, entre autre, visé la gare d'Ottignies et la gare de triage, mais la ville et les campagnes ont également souffert de ces bombardements. Site du CEGES (Centre d'Études et de Documentation Guerre et Sociétés contemporaines), consulté le 2 mars 2011 : http://www.cegesoma.be/cms/catalogue_fr.php. 
de maltraitance (trois femmes et deux hommes). Par maltraitance, nous entendons l'exploitation physique, la malnutrition et les violences physiques allant jusqu'à l'abus sexuel. Toutes ces situations de nonrespect de l'enfant ont laissé des marques profondes. L'enfant juif caché se retrouvait dans l'incapacité de se rebeller car leur survie dépendait des personnes qui le cachaient. Souvent, ces milieux maltraitants accueillaient les enfants davantage dans un but financier et ne se souciaient pas de leur bien-être. Certains enfants ont été exploités ou mal nourris. Dans ces situations, l'enfant juif était souvent traité différemment des autres membres de la famille. Madame O. souligne : «J'étais affamée [...] et j'étais très malheureuse parce qu'on nourrissait les enfants de la famille le soir et moi, on m'envoyait au lit avant. » Deux enfants ont été retirés du milieu d'accueil par leurs parents, mais les autres sont restés près de deux ans dans le milieu maltraitant : " J'ai été à plusieurs reprises enfermé dans une cave noire. On me disait pour que j'aie bien peur qu'il y avait des souris et des rats dans la cave, j'étais terrorisé. Je hurlais tout le temps quand j'étais dans cette cave. Et donc, je me suis remis à faire pipi au lit... Alors, quand mes parents sont venus me rendre visite, ils se sont rendu compte de la catastrophe. C'était en train de me détruire évidemment. À ce moment-là, ils m'ont sorti de là, mais je crois que le mal était fait... » (Monsieur T., né en 1936).

Ces comportements maltraitants ont laissé des traces importantes dans l'organisation psychique d'un enfant menacé de mort, vulnérable et déjà fragilisé par les persécutions. La peur d'être découvert par les Allemands, la séparation des parents, les comportements de maltraitance et l'impossibilité de retrouver une sécurité ont eu des répercussions catastrophiques chez cet enfant, dont les traces demeurent toujours visibles à l'heure actuelle: "Comme vous voyez, j'ai encore certains tics faciaux. Quand je suis sorti de la guerre, j'avais énormément de tics, j'avais beaucoup de troubles psychologiques. Les psychiatres à l'époque, craignaient très, très fort pour moi. Ils estimaient que j'étais vraiment très, très durement marqué par toute la peur que j'ai eue... » Monsieur F. décrit lui aussi plusieurs faits de maltraitance physique au sein d'une institution catholique. Plusieurs femmes ont également vécu dans un climat malsain (risque d'abus sexuels), et deux d'entre elles ont subi des violences sexuelles. Dans 
notre recherche, nous avons constaté que le placement dans un milieu d'accueil favorable a souvent permis à l'enfant de se sentir davantage protégé et d'atténuer le sentiment d'exclusion. Au contraire, le placement dans un milieu maltraitant a souvent détruit la représentation interne d'un environnement protecteur, induit un sentiment d'insécurité très fort et favorisé un comportement de méfiance encore plus important chez l'enfant.

De nombreux enfants juifs cachés se souviennent du retour des survivants des camps de la mort comme un événement effrayant, presque irréel. Monsieur A., né en 1935, se souvient avoir vu ses oncles rentrer des camps : "Mon oncle a réussi à se sortir de plusieurs camps et il a travaillé, et alors voilà il s'en est sorti. Et alors, j'en ai un autre qui s'en est sorti aussi. Et donc, j'ai vu revenir ces gens dans les années 45-46 dans des états... Je les ai vus, comme on peut les voir au cinéma. Enfin, je ne les voyais pas au cinéma, mais moi, je les ai vus quand ils sont revenus à Bruxelles. »

Les enfants ont été confrontés à un « réel terrifiant $»^{38}$. Pour ceux qui retrouvent un parent survivant des camps, l'image souvent idéalisée du parent s'effondre, rendant le processus d'identification et de « réappropriation » de l'image parentale difficile : « Ça a été le choc. En arrivant, j'ai vu quelqu'un, comme j'étais incapable de reconnaître que c'était mon père. J'ai vu un vieillard, c'est tout. Ça m'a... J'étais plutôt étonné qu'on m'ait dit que c'était mon père. Il m'a fallu un bon moment pour que je m'habitue à l'idée que ça a été mon père $[\ldots]$ il avait l'air d'être 30 ans plus vieux que ma mère. Quand ils sont arrivés, ils étaient des vieillards, ils étaient... je ne sais pas... $38,40 \mathrm{~kg}$. C'était des ruines quoi! Alors, on nous présente ça, avec des yeux exorbités, pas beaux du tout... Plus effrayants qu'autre chose. J'étais plutôt effrayé, je me demandais ce que j'allais faire avec ce monsieur. » (Monsieur T., né en 1936).

L'après-guerre est lié à l'apparition de nouvelles souffrances et à la découverte du massacre : "Quand on est sorti vivant de tout ça, ce n'était pas une fin en soi, on commençait seulement à découvrir l'ampleur du désastre » (Madame R.). La révélation des atrocités commises durant la Shoah a parfois été vécue de façon extrêmement

${ }^{38} \mathrm{~J}$. Altounian, op. cit. 
brutale par les enfants. Madame Ch., orpheline, fut forcée de prendre conscience du destin tragique de ses parents et fut, de ce fait, confrontée à un réel effrayant : " Il y avait une exposition de photos, organisée par l'armée américaine, sur la découverte des camps de concentration. C'étaient de grandes photos très réalistes et l'exposition était interdite aux moins de 16 ans, à juste titre. Mais ma grand-mère qui voulait absolument que je voie ça, m'a fait entrer par la sortie. J'avais 11 ou 12 ans. C'est vous dire comme j'ai été évidemment très frappée. Elle voulait, il fallait que je sache. Je lui en ai voulu. Et là, j'ai eu des cauchemars après. C'était des photos de charniers, de baraquements, des photos de prisonniers derrière des barbelés, des photos prises, enfin bon... »

Ces prises de conscience ont été terrifiantes pour l'enfant. Plusieurs personnes soulignent avoir été violemment obligées de se rendre compte du destin tragique de leurs proches. Cette confrontation au réel de la mort a eu un impact déstructurant et anéantissant pour la plupart des sujets, détruisant également l'image idéalisée des parents. Paradoxalement, alors que ces scènes terrifiantes enlèvent à l'enfant tout espoir de retrouver ses parents, le fait de continuer à espérer un retour impossible représente souvent l'unique possibilité de survie. Cette protection vitale est d'autant plus présente chez les plus jeunes que la perte a eu lieu de façon précoce et qu'ils pouvaient difficilement conceptualiser la mort.

Lorsque les enfants ont retrouvé un ou leurs deux parents, ces retrouvailles ont souvent été traumatiques, bien que leur souffrance n'ait pas été reconnue comme telle par les adultes. D'une part, parce que l'enfant fut forcé de se séparer du milieu d'accueil auquel il s'était adapté, et d'autre part parce que les parents étaient fragilisés et plus vulnérables. Face à des parents détruits, certains enfants ont idéalisé leur famille d'accueil et n'ont pas accepté de retourner dans leur famille d'origine. De plus, une rupture affective s'est souvent installée entre les enfants et les parents en raison de la séparation précoce, et ce parfois malgré les tentatives des parents pour renouer le lien. Par ailleurs, lorsqu'un parent avait survécu, celui-ci devait faire face à la perte du conjoint et était moins disponible pour l'enfant. Souvent, l'enfant n'a pas retrouvé le parent tel qu'il l'avait connu avant la guerre. Par exemple, madame S., née en 1935, a retrouvé ses deux 
parents. Sa mère était dans la Résistance pendant la guerre, tandis que son père est revenu des camps de concentration. Elle souligne qu'après la guerre, elle a retrouvé une mère qui était devenue « malléable », « en liquéfaction », comme si « elle avait perdu son âme dans cette guerre ». Son père, rescapé d'Auschwitz, est revenu détruit par l'expérience concentrationnaire. "Quand il est revenu, c'était un autre homme. Il était d'une violence incroyable », dit-elle. Après la guerre, elle a dû continuer à se construire malgré la violence, les insultes, le harcèlement moral, et la destruction de l'estime de soi : « Il m'a fait porter un poids qui n'était pas le mien et [elle renifle]. Ça a vraiment été très loin [rires]. Il me cavalait derrière avec un couteau de cuisine mais je courais plus vite [rires]. Maintenant j'en ris mais c'est parce que... parce que je ne voulais pas accepter d'être femme d'ouvrage et je lui disais: "Je ferai autre chose". "Eh bien alors, tu ne peux faire que le trottoir !", "Eh bien, j’irai faire le trottoir !" Et ça, ça déclenchait chez lui une... une espèce de rage meurtrière. Vraiment, une rage. Je suppose qu'à Auschwitz, il a souffert l'indicible et que quelqu'un ose après qu'il ait souffert tout ça... »

Après la guerre, certains parents étaient devenus incapables de s'occuper de leurs enfants. Les problèmes de santé, la perte des proches et la difficile reconstruction des parents après la guerre ont parfois amené certains enfants à assumer des tâches trop importantes pour leur âge. Madame R. a retrouvé ses parents, mais a dû assumer d'importantes responsabilités familiales suite aux problèmes de santé de sa mère : "On l'a mise dans un sanatorium. J'avais 12 ans. Ma mère, je venais de la retrouver et je la reperds. Je dois m'occuper de mon frère et de mon père, je dois m'occuper de l'école, je dois faire les courses. J'étais plongée directement dans les jours qui ont suivi la Libération dans une vie d'adulte qui n'était que le prolongement de ce que je venais de vivre sauf que je pouvais marcher dans la rue. Ça, c'est aussi une des conséquences. »

Dans ce cas-ci, malgré les retrouvailles, les figures parentales n'étaient plus disponibles pour l'enfant. Alors qu'elle avait besoin de retrouver des parents qui prennent soin d'elle après une longue séparation, madame R. s'est retrouvée face à des parents incapables d'assumer leurs fonctions parentales : « J'ai remarqué que mes parents ont baissé les bras devant l'éducation de leurs enfants à ce moment-là 
parce qu'ils étaient écrasés par le poids de la douleur. Ils avaient autre chose à faire. " Cette situation a renforcé la solitude de l'enfant et la croyance en l'impossibilité de compter sur les figures parentales. La mère de madame $\mathrm{S}$. était elle aussi devenue incapable de répondre aux besoins primaires de ses enfants, laissant presque mourir son troisième enfant de déshydratation après la guerre : « Je me souviens, une fois, ma sœur avait moins d'un an, j'ai téléphoné aux urgences parce que je suis passée par là et je voyais que cet enfant était mourant, et ma mère ne se rendait compte de rien! » Les dégâts provoqués par la Shoah ont perduré bien au-delà de la période de guerre ${ }^{39}$.

\section{Les souvenirs liés aux séparations et aux pertes}

Parmi les souvenirs liés aux pertes, nous avons dégagé les expériences de séparation, l'angoisse répétitive de perdre ses parents, l'arrestation des parents, l'interdiction de maintenir des liens avec la famille d'accueil, la perte des proches et la spoliation des biens.

Les séparations représentent un traumatisme central dans l'expérience des enfants juifs cachés. Dans notre population, $95 \%$ des anciens enfants juifs cachés (38 personnes sur 40) ont été séparés de leurs parents. La séparation suscite encore beaucoup d'émotions au moment du récit. Cet événement a suscité un premier choc pour l'enfant. Souvent, il ne comprenait pas les raisons de la séparation et de son placement. Les enfants ont souvent manifesté leur détresse (perte d'appétit, pleurs, énurésie). Les séparations avaient lieu dans un climat d'angoisse, de non-préparation et de silence. De plus, l'enfant ne connaissait souvent ni l'endroit, ni les personnes chez qui il était caché. La plupart des enfants juifs cachés se sont sentis abandonnés par les parents ${ }^{40}$. Nombreux sont ceux qui font également état d'un immense manque de tendresse, qui n'a parfois pas pu être comblé par le milieu d'accueil : «Avec un enfant de 5 ans, on doit quand même un peu... Vous savez, quand on n'est plus avec sa mère, on manque d'affection. Et elle [la mère d'accueil] ne savait pas donner de l'affection. Elle ne

${ }^{39}$ Y. Gampel, Ces parents qui vivent à travers moi. Les enfants des guerres, Paris, 2005.

${ }^{40}$ M. Frydman, op. cit. ; C. H. Haber, « The analysis of a latency-age survivor of the Holocaust », dans Psychoanalytic Review, 75 (4), 1988, pp. 641-651 ; C. Vegh, Je ne lui ai pas dit au revoir. Des enfants de déportés parlent, Paris, 1979. 
savait pas... » (Madame Fo., née en 1937); « Les religieuses faisaient tout le possible pour nous, mais il nous manquait la tendresse d'une maman... [très émue] » (Madame Sch., née en 1931). Contrairement à ces situations, d'autres enfants ont reçu l'attention et l'affection dont ils avaient besoin dans la famille d'accueil : "C'était ma seconde mère. » (Madame Ch., née en 1935); « Je peux considérer dans l'aprèscoup que lui était comme un père substitutif et elle aussi comme une mère substitutive » (Monsieur A., né en 1935).

Néanmoins, la majorité des enfants juifs cachés s'est sentie abandonnée par ses parents ${ }^{41}$. En réponse à l'impression d'abandon, de nombreux enfants ont également éprouvé un sentiment de colère, souvent indicible et inconscient, envers leurs parents.

Certains enfants ont également été séparés d'objets symboliques et rassurants, qui les reliaient à leurs parents et au monde qui leur était familier : " J'ai toujours essayé de retrouver cette même poupée que j'ai laissée chez ma mère quand je suis partie. J'ai dû abandonner ma poupée. Et c'est vrai que les gens chez qui j'étais cachée, je me suis sauvée de là quand j'avais 6 ans, je me suis sauvée, et j'ai retrouvé ma maison et il y avait des scellés aux portes que les Allemands avaient mis, et j'ai tambouriné parce que je voulais récupérer ma poupée... » (Madame C., née en 1936).

Généralement, l'éloignement de la fratrie fut peu évoqué dans les récits, sauf lorsque les enfants furent d'abord cachés ensemble, puis séparés. La présence d'un frère ou d'une sœur rendait alors la séparation d'avec les parents moins traumatique car les enfants se rassuraient mutuellement. Madame $F$. se souvient avec douleur de la séparation d'avec son frère : " Je me souviens encore comme si c'était hier, on était au pont du canal et puis maman nous a dit: "Vous n'allez pas en pensionnat. On vous a trouvé une place à tous les deux". On s'est mis à hurler, on a hurlé, on a pleuré. ». C'est souvent à ce moment-là que la perte des parents fut douloureusement ressentie: « Je ne savais pas manger, je pleurais des nuits entières dans mon lit après mes parents, après papa et maman. Je n'ai jamais su comprendre. "

${ }^{41}$ C. Vegh, op. cit.; C. H. Haber, op. cit.; M. Frydman, op. cit.; A. Fohn, « Secret et traumatisme... », op. cit. 
L'angoisse répétitive quant au sort des parents était également particulièrement présente chez les enfants qui étaient conscients du danger. La séparation et l'incompréhension ont souvent entraîné une inquiétude et un questionnement important vis-à-vis du sort de leurs parents. Où sont-ils ? Sont-ils toujours en vie ? Est-ce que je vais les revoir? Chez madame B., cette angoisse était d'autant plus forte qu'elle voyait ses parents pendant la guerre dans un climat d'insécurité. Chez elle, la crainte de les perdre était permanente : « Mes parents avaient fourni à l'institut un certificat médical comme quoi il était indispensable psychologiquement que je retourne toutes les semaines chez eux. Avant de rentrer, je devais toujours faire une halte dans une papeterie de la rue de la Victoire pour demander si tout allait bien et si je pouvais rentrer, si je pouvais y aller ou s'il y avait eu malheur. Bon je vivais ça dans la peur du fait que je doive aller demander si tout allait bien. Ça avait créé un climat d'inquiétude en moi vraiment très fort. »

L'arrestation des parents représente également une expérience douloureuse, souvent suivie du non-retour des parents après la guerre. Quelques rares enfants ont été directement alertés de l'arrestation de leurs parents pendant la guerre : « La femme de ménage est venue un soir toute agitée pour me dire que mes parents avaient été arrêtés. Elle savait où j'étais. Elle avait compris que c'était les Allemands qui étaient venus les arrêter. Elle avait retiré de l'appartement tout ce qui pouvait faire penser qu'il y avait un enfant : toutes les photos, les papiers. Elle nous les apportait et disait : "Attention, peut-être qu'ils vont venir chercher la petite, il faut la cacher !"» (Madame Ch., née en 1935).

Dans son récit, le vécu subjectif est absent lorsqu'elle évoque cet événement. Seuls les faits et le changement de nom sont mis en évidence. Il est possible que l'impact traumatique ait engendré une « sidération» psychique qui l'empêchait de penser et d'élaborer ces événements ${ }^{42}$. Actuellement, madame Ch. écrit l'histoire de sa vie, processus qui se révèle douloureux, mais qui semble l'aider à élaborer son vécu et la perte de ses parents.

${ }^{42}$ S. Ferenczi, Journal clinique (janvier-octobre 1932), Paris, 1985. 
Certains enfants ont également vu leurs parents se faire arrêter. Dans son récit, monsieur Su. évoque cette situation douloureuse. Âgé de 6 ans, il avait quitté la ville d'Anvers avec ses parents pour se cacher à la campagne. Pour échapper aux rafles, ses parents se cachaient dans le cimetière situé en face de la maison où étaient les enfants. Suite à une dénonciation, ses parents ont été arrêtés : «Ma sœur et moi, nous vivions dans un grenier. On ne pouvait pas sortir, on était vraiment enfermé, on n'avait qu'une petite lucarne, une petite fenêtre où on pouvait regarder dehors. C'est par cette fenêtre là que j'ai vu... ma sœur et moi nous étions restés en haut, qu'on a vu les Allemands emmener mes parents en camion, en camion allemand, et depuis lors on ne les a plus jamais revus. Alors, ma sœur et moi, nous avons été séparés après le départ de mes parents. »

Ce souvenir est gravé dans la mémoire de monsieur Su. Après la guerre, il ne retrouva aucun proche survivant, excepté sa grand-mère maternelle. Ses parents ont été déportés à Auschwitz, tandis que ses grands-parents paternels et sa sœur sont décédés dans un bombardement. Il ne parle que très peu de son histoire. Il se sent d'ailleurs coupable de ne pas pouvoir en parler plus souvent (« Je sais que je suis fautif parce qu'il faut parler $»)$. Sa souffrance est encore très présente. Durant les entretiens, il est néanmoins parvenu à raconter son histoire et s'est senti soulagé d'avoir pu en parler. Il désire davantage la partager avec ses enfants et ses petits-enfants.

La séparation du milieu d'accueil fut souvent évoquée dans les récits et représente un événement douloureux, particulièrement chez les plus jeunes et lorsque l'enfant s'était attaché au milieu d'accueil. Ce nouvel éloignement, renvoyant à celui d'avec les parents, a souvent fragilisé les enfants juifs cachés ${ }^{43}$ : « Je pleurais toutes les larmes de mon corps, je voulais retourner là-bas [dans la famille d'accueil]. Le traumatisme pour moi, que du contraire... Le traumatisme, pour moi, c'était plutôt quand je rentrais dans ma famille. » (Monsieur Ba., né en 1940)

Nous pouvons dire qu'il s'agit d'un traumatisme en après-coup, c'est-à-dire un traumatisme composé de deux temps ${ }^{44}$. Dans un

${ }^{43}$ A. Fohn - S. Heenen-Wolff, « The Destiny of an Unacknowledged Trauma... », op. cit.

${ }^{44}$ S. Freud, « Esquisse d'une psychologie scientifique », dans S. Freud (éd., 1956), La naissance de la psychanalyse : lettres à Wilhelm Fliess, Paris, 1895, pp. 307-396 ; J. Laplanche, Problématiques VI. L'après-coup, Paris, 2006. 
premier temps, le sujet vit un événement potentiellement traumatique (ici, la première séparation) qui reste à l'état de latence. Ce n'est que dans un deuxième temps, lorsque le sujet est confronté à un autre événement plus tardif (la deuxième séparation) qui vient réveiller le souvenir de l'événement antérieur, que l'impact traumatique se déploie. Après la guerre, l'enfant fut, une fois de plus, séparé d'un milieu auquel il s'était adapté, et ce sans préparation psychique aucune et sans que l'adaptation ne se fasse progressivement.

Un nombre non négligeable d'enfants juifs cachés ont évoqué la violence de certaines séparations d'avec le milieu d'accueil. C'est particulièrement le cas des orphelins et de ceux qui ont perdu un parent. Ses parents n'ayant pas survécu à la Shoah, monsieur N. a continué à vivre dans sa famille d'accueil après la guerre. Après avoir vécu six années à leurs côtés, il fut placé du jour au lendemain par son oncle dans un home de l'AIVG (Aide aux Israélites Victimes de la Guerre), sans aucune préparation psychique possible ${ }^{45}$. Cette séparation, une fois de plus très brutale, a généré une souffrance importante et un sentiment d'impuissance, anéantissant une fois de plus la « continuité d'être » de l'enfant ${ }^{46}$. Certains milieux d'accueil ont également refusé de rendre l'enfant à sa famille : « Mon père a retrouvé notre trace, là où nous étions, et il est venu. Ils n'ont pas voulu que nous ayons un contact avec lui. Ils n'ont surtout pas voulu nous rendre à la Libération. Un jour, c'était en 44-45, mon père est venu nous enlever à la sortie de l'école. Mon père est venu, il nous a kidnappés parce que les personnes ne voulaient pas nous rendre. » (Madame Ro., née en 1937).

Madame C., née en 1936, a vécu une situation similaire. Alors que sa mère et sa sœur ont péri dans la Shoah, son père, survivant des

\footnotetext{
${ }^{45}$ Après la guerre, les homes qui appartenaient à l'Association des Juifs en Belgique (AJB) ont été repris par l'Aide aux Israélites Victimes de la Guerre (AIVG). Par ailleurs, de nouvelles structure ont été ouvertes pour accueillir les enfants orphelins afin qu'ils puissent se reconstruire et continuer à grandir dans un milieu juif (Voir V. Teitelbaum-Hirsch, op. cit.) Pour plus d'informations concernant l'AIVG et ses homes, voir C. Massange, Bâtir le lendemain. L'Aide aux Israélites Victimes de la Guerre et le Service Social Juif de 1944 à nos jours, Bruxelles, 2002 ; Ead. « De l'Aide aux Israélites Victimes de la Guerre au Service Social Juif. De 1944 à nos jours : 55 ans d'histoire des Juifs en Belgique », dans Les Cahiers de la Mémoire contemporaine, ${ }^{\circ}{ }^{1,} 1999$ pp. 157-167 ; Ead., « Hirondelles et Aiglons. Les adolescents de l'AIVG », dans Les Cahiers de la Mémoire Contemporaine, $n^{\circ} 3,2001$, pp. 175-212.

${ }^{46}$ D. W. Winnicott, De la pédiatrie à la psychanalyse, éd. 1969, Paris, 1958.
} 
camps, s'est vu confronté à un refus de la famille d'accueil de lui rendre son enfant : «On me cachait dans un placard en me disant : "Tu sais, ce n'est pas ton père. Tu es une enfant volée. Cache-toi !" Alors on me cachait dans le fond d'un placard. Ce n'est pas pour rien que j'ai des angoisses maintenant... »

Ces situations ont créé une confusion dans le psychisme de l'enfant. Ici, l'enfant a cru à l'histoire qui lui avait été racontée par la famille d'accueil et ne voulait dès lors plus retourner vivre avec son père : « Une fois, il est venu et là, on m'a dit : "C'est ton père". Mais moi j'ai dit: "Non, ce n'est pas mon père!" Pour moi, j’étais une enfant volée... » Certaines institutions catholiques se sont également opposées à rendre les enfants après la guerre. En France, l'histoire des frères Finaly fut fortement médiatisée ${ }^{47}$. Lorsque la communauté juive a retrouvé la trace de ces orphelins quelques années plus tard, les personnes qui les ont cachés se sont opposées à leur départ. Katy Hazan relève également le fait que ces situations étaient souvent dramatiques lorsque le milieu d'accueil ne voulait pas rendre les enfants par conviction religieuse ou parce qu'il s'y était attaché ${ }^{48}$. En Belgique, nous avons rencontré deux sœurs qui ont vécu une expérience similaire. Cachées dans une crèche à l'âge d'un et deux ans et orphelines, personne ne vint les réclamer après la guerre. Ignorant tout de leur passé et de leurs origines juives, les enfants ont été élevées par les sœurs catholiques qui les avaient cachées durant la guerre. Lorsque plusieurs années plus tard, une organisation juive retrouva leur trace, les religieuses ont empêché leur départ: « Du jour au lendemain, j’ai dû aller en pension à Tournai et ça c'était terriblement dramatique. [...] Et alors là, je n'ai pas changé de nom de famille. Apparemment, les pensionnaires ne pouvaient pas soulever le problème juif parce que ça allait très fort me heurter. Et donc on n’en

${ }^{47}$ Robert et Gérald Finaly, nés de parents juifs autrichiens établis en France, ont été confiés à des sœurs catholiques durant la Seconde Guerre mondiale alors qu'ils étaient âgés de 2 et 3 ans. Placés ensuite dans une crèche, ils n'ont pas été récupérés par leurs parents, déportés à Auschwitz. La directrice catholique ne voulait pas les rendre aux survivants de leur famille. Il fallut attendre 1953 pour que les enfants rejoignent leur famille en Israël. Voir C. Poujol, Les enfants cachés. L'affaire Finaly (1945-1953), Paris, 2006.

${ }^{48}$ K. Hazan, « Enfants cachés, enfants retrouvés », dans Les Cahiers de la Shoah, n 1(9), 2007, pp. 181-212. 
parlait jamais, j'ai vraiment occulté toute ma jeunesse. » (Madame G., née en 1939)

Ces séparations brutales et la rupture avec le milieu juif ont souvent été désastreuses à long terme pour la construction des sujets et le développement de leur vie affective. Leur vécu et leurs souffrances qui y étaient liées n'ont absolument pas été pris en compte par les adultes. Madame G., orpheline, n'a pas pu s'appuyer sur ses parents pour faire face à cette situation et se réinsérer dans le milieu juif. Elle est partie à l'étranger pour tenter de se reconstruire et mettre son passé à distance : «Je suis partie aux États-Unis aussi peut-être pour cacher ce que j'étais et deuxièmement aussi pour devenir quelqu'un. » Actuellement, les souffrances liées à l'identité et à la perte de ses parents sont toujours très présentes chez madame $G$.

Après la guerre, certains enfants se sont également trouvés face à une interdiction de maintenir des liens avec leur famille d'accueil. Or, tous les enfants qui s'étaient attachés à une famille ont exprimé le besoin d'entretenir des contacts. Cette interdiction a souvent eu des conséquences dramatiques pour l'enfant. Nous constatons d'ailleurs que le maintien des liens avec la famille d'accueil a souvent permis d'atténuer le choc lié à la séparation et de restaurer un sentiment de continuité, déjà mis à mal lors de la première séparation. Au contraire, l'interdiction du maintien des liens avec cette famille a une fois de plus brisé la "continuité d'être" du sujet ${ }^{49}$ : « Ça m'a fort fait souffrir parce que j'avais pour elle [mère d'accueil] non seulement de la reconnaissance, mais une énorme amitié. C'était ma seconde mère. Je la connaissais depuis que j'étais bébé. C'est le plus sale coup que ma famille m'ait fait, c'était me couper de mes vraies racines. Mes vraies racines, ce n'étaient pas eux, c'était elle. » (Madame Ch., née en 1935).

Cette interdiction fut vécue comme une terrible injustice contre laquelle elle s'est révoltée. Nous constatons que ceci a souvent amené l'enfant à s'opposer et à rejeter sa famille, et parfois bien au-delà, son lien à la judéité.

Par ailleurs, les enfants juifs cachés, comme tous les autres survivants de la Shoah, ont dû faire face à de nombreuses pertes (parents, grands-parents, frères, sœurs, cousins, oncles, tantes). La

${ }^{49}$ D. W. Winnicott, op. cit. 
perte la plus significative reste celle de leur(s) parent(s). En tant qu'enfants, ils avaient encore besoin d'eux pour grandir. La disparition d'un ou des deux parents fut souvent catastrophique pour l'enfant, et le deuil impossible : «Alors vous savez... Le plus dur... c'est que ma maman a été déportée... [pleurs] Et qu'elle n'est jamais revenue... euh... des camps de concentration... C'est terrible... Vous savez... Pourtant, j'ai fait une psychanalyse... Mais c'est terrible pour un enfant... Un enfant, il a besoin... C'est terrible. » (Madame Ga., née en 1931).

La souffrance des orphelins et des enfants qui ont perdu un parent est très importante. Ils relatent souvent des difficultés relationnelles, affectives et psychiques plus importantes à long terme (sentiments de perte, d'abandon et de solitude plus forts, plus d'épisodes dépressifs, manque d'appartenance) par rapport aux enfants qui ont retrouvé leurs deux parents. Par ailleurs, ceux qui ont perdu un parent dans la Shoah sont souvent retournés vivre avec le parent survivant, parfois rescapé des camps, et souvent profondément affecté par la perte de son conjoint : "Mon père, il était toujours déprimé. Il m’en parlait toujours à moi, il me parlait des camps de concentration, ce qui était une enfance horrible parce que c'est trop lourd pour moi. Et je l'ai dit à mon père plus tard, beaucoup plus tard. Et il me dit : "Je ne voulais pas que vous oubliiez votre mère." Comme si on pouvait l'oublier... » (Madame Ga., née en 1931).

Ces enfants ont souvent terriblement souffert de cette situation, portant le poids de leur propre douleur et celle du parent survivant. Le placement dans une institution juive après la guerre a pu alléger le poids qu'ils auraient porté dans un milieu familial dévasté. De plus, ce placement leur a dans bien des cas permis de se reconstruire psychologiquement : "Je suis resté dans les homes de l'AIVG jusqu'à mes 18 ans au moment où je suis rentré à l'université. Je suis resté dans des homes où nous étions très bien et j'en garde un très bon souvenir. Là, nous avons réappris à revivre... » (Monsieur F., né en 1936, a perdu son père dans la Shoah).

La majorité des enfants juifs cachés interviewés ont également perdu leurs grands-parents dans la Shoah. La disparition de cette génération les marque particulièrement dans l'après-coup. En devenant des grands-parents et en découvrant le bonheur d'avoir des 
petits-enfants, ils se sont souvent rendu compte à ce moment-là de ce dont ils ont été privés : «C'est en devenant grand-mère que je me suis rendu compte que je n'avais jamais vu mes grands-parents. C'est peutêtre pour ça que je suis une grand-mère poule... Je n'ai pas vu une photo. Je ne sais pas ce que c'est que d'être grands-parents. C'est une très grande peine. Mes petites-filles ont 22 et 18 ans [émue]. »

La spoliation des biens (résidences, argent, bijoux, meubles, vêtements, photos) représente également un élément important de leur histoire, les biens ayant été emportés et volés par les nazis ou même parfois par leurs voisins. Après la guerre, il s'agissait de tout reconstruire : "On a démarré avec rien, pas d'héritage. Quand mes parents sont ressortis, dans leur appartement, il n'y avait plus rien... Tout avait été nettoyé, spolié, vidé, il ne restait rien... Il fallait tout recommencer à zéro. » (Monsieur B.).

Les orphelins n'ont souvent pas pu conserver une photo de leur famille. Ce n'est parfois que très récemment que certains ont redécouvert le visage de leurs parents : «Moi, j'ai fait ça il y a 2 ans. J'y ai vu pour la première fois mon père en photo, j'ai dû attendre 72 ans pour voir le visage de mon père $»^{50}$ (Monsieur S., né en 1933). Les recherches personnelles et un retour sur les traces de leur passé les ont souvent aidés à renouer avec le passé et à élaborer les chocs vécus, voire parfois à entamer un processus de deuil, longtemps resté en suspens ${ }^{51}$.

\section{Discussion}

L'analyse des récits de vie d'anciens enfants juifs cachés nous a permis de dégager de nombreux souvenirs traumatiques, vestiges mnésiques d'un passé douloureux. La précision et la longévité de ces souvenirs montrent à quel point ils ont conservé des traces durables des événements passés, contrairement aux idées et croyances collectives de l'époque (ils étaient « chanceux » et « trop jeunes pour se souvenir »). Sur base de notre analyse, nous avons pu relever de

\footnotetext{
50 Notamment par l'intermédiaire de l'Office des Étrangers, chargé de gérer l'accès des étrangers au territoire, leur séjour et leur établissement.

${ }^{51}$ Y. Mouchenik, « De la disparition au deuil chez les orphelins de la Shoah », dans L'autre, Cliniques, Cultures et Sociétés, $\mathbf{n}^{\circ} 5$ (1), 2004, pp. 129-141.
} 
nombreuses réminiscences liées à leur enfance, et ce même chez de très jeunes enfants. Afin de présenter nos résultats de façon structurée, nous avons regroupé ces souvenirs en quatre catégories, à savoir ceux relatifs à l'atteinte identitaire, à la crainte d'être découvert, à la violence et aux pertes. Chaque enfant juif caché a souvent vécu au moins un événement de chaque catégorie.

Ces souvenirs, traduits en récits, nous ont permis d'appréhender la réalité subjective de nombreux enfants juifs cachés et les événements traumatiques multiples auxquels ils ont été confrontés durant la guerre et dans l'immédiat après-guerre. Ces expériences précoces et douloureuses ont souvent façonné leur manière d'être, de vivre et de réagir à d'autres événements ultérieurs. Le recul rétrospectif de ces jeunes survivants devenus adultes et l'analyse personnelle de leur passé confèrent un poids aux récits qui se révèlent être d'une richesse inouïe. La plupart des sujets font eux-mêmes beaucoup de liens entre passé, présent, futur et leurs difficultés personnelles, leurs valeurs, leur combat pour lutter contre l'injustice et l'exclusion. Au-delà d'une expérience individuelle, la Shoah représente une expérience collective qui marque à jamais la communauté juive, les survivants et les générations suivantes. Étant donné la puissance du traumatisme, les survivants ont été incapables d'intégrer à eux seuls les événements auxquels ils ont dû faire face. Comme le souligne Ciccone, « on ne peut pas penser tout seul; pour penser, on a besoin d'un autre, et d'un autre qui nous investisse. [...] On ne peut comprendre, symboliser, représenter qu'avec l'aide d'un autre, d'abord au-dehors de soi, puis intériorisé, mis au-dedans de soi, constitué comme objet interne ${ }^{52}$. Ce processus a souvent fait défaut chez les enfants juifs cachés car, à ce moment-là, "l'Autre", l'adulte rassurant, n'existait pas ou n'était pas disponible pour aider l'enfant à intégrer ces expériences traumatiques. L'adulte était lui-même marqué par son vécu et ne se rendait pas compte des souffrances de l'enfant, d'autant plus que celui-ci avait appris à se taire. L'expérience des enfants juifs cachés nous montre que c'est à travers le social (par exemple la reconnaissance sociale de leurs souffrances, les rassemblements de pairs, les commémorations, les

${ }^{52}$ A. Ciccone, «L'éclosion de la vie psychique », dans A. Ciccone - Y. Gauthier - B. Golse - D. Stern (éds.), Naissance et développement de la vie psychique, Ramonville Saint-Agne, 2008, pp. 11 37. Citation p. 12. 
groupes de parole) et les générations futures, particulièrement par l'intermédiaire de leurs petits-enfants, qu'ils ont pu commencer à élaborer et intégrer leur propre vécu. D’autres facteurs personnels tels que le vieillissement, la retraite, le besoin pressant de connaître le passé et de le transmettre, les ont poussés à s'interroger, à affronter le passé et à en parler. La démarche du récit de vie avec d'anciens enfants juifs cachés s'inscrit dans cette même dynamique, c'est-à-dire qu'elle favorise une reconnaissance sociale et émerge d'un désir, d'un besoin personnel des survivants.

Les souvenirs autobiographiques, revécus et racontés dans une relation basée sur l'écoute et l'attention, ont permis de nommer et de décrire un passé douloureux. Pour certains, il s'agissait d'une première prise de parole, pour d'autres, il s'agissait d'une narration plus approfondie avec un tiers, extérieur à la famille, qu'ils craignaient moins de blesser et de «traumatiser ». Ce besoin de partager leur histoire est encore très présent pour de nombreuses personnes. Monsieur P. a souligné : « Je pense qu’inconsciemment, j’ai attendu toute ma vie votre visite pour faire mon histoire. » Le récit de vie lui a donné un cadre possible pour élaborer son vécu, pour donner une place à l'émotionnel souvent très retenu dans sa vie quotidienne. Sa souffrance pouvait s'exprimer dans le lien à l'autre et était reconnue, entendue. Tout comme Herman Vandormael, nous avons constaté que « le choc psychologique du relâchement brutal du traumatisme, après tant d'années de silence, a montré l'importance de la charge émotionnelle encore présente, au point que de nombreuses personnes ne pouvaient contenir leurs larmes lorsqu'elles étaient confrontées à leurs souvenirs ${ }^{53}$. Toutefois, une fois les mots posés, le traumatisme n'a plus le même impact mortifère pour autant qu'il puisse être élaboré et symbolisé. Comme le souligne Altounian, la narration en tant que mise en mots du massacre collectif permet aux survivants de sortir de l'isolement dans lequel ils sont emprisonnés, de se séparer des défunts et de se détacher de l'emprise traumatique: «Mettre en mots le meurtre silencieux [...] c'est certes mettre en terre leurs ancêtres, mais c'est surtout pouvoir enfin se démettre de l'emprise du crime. " $^{54}$ Le récit de vie permet aussi souvent une réappropriation de l'histoire

${ }^{53}$ H. Vandormael, Les enfants cachés se souviennent, Bruxelles, 2010.

${ }^{54}$ J. Altounian, op. cit., p. 2. 
personnelle qui fait partie du processus de guérison. Revivre son histoire et les émotions qui y sont associées permet de se détacher de l'emprise mortifère et d'entamer un travail de deuil. Eva Metzger Brown, une ancienne enfant juive cachée, expliquait : "Une fois que j'ai été capable de ressentir mon histoire réellement comme "mienne", j'étais capable de commencer à faire le deuil de mes propres pertes et de guérir mes blessures profondément ressenties à l'intérieur de mon être. $\|^{55}$ Golse précise encore que le témoignage peut intervenir comme un élément susceptible d'inscrire le traumatisme du côté de la vie ${ }^{56}$. Tout comme les recherches personnelles permettent aux anciens enfants juifs d'assembler les pièces du puzzle, le récit de vie leur a permis de retracer de façon continue une histoire jusque-là souvent fragmentée, sinon éparpillée.

${ }^{55}$ E. Metzger Brown, « Recovering Memory », dans Lilith Magazine, ${ }^{\circ} 23$ (3), 1998, pp. 16-17. Citation p. 17.

${ }^{56}$ B. Golse, «Trauma et après-coup », dans K. Nissikas (éd.), Le trauma entre création et destruction, Paris, 2004, pp. 91-110. 